EPJ Web of Conferences 13, 06004 (2011)

DOI: $10.1051 /$ epjconf/20111306004

(c) Owned by the authors, published by EDP Sciences, 2011

\title{
Transport coefficients in non-quasiparticle systems
}

\author{
Antal Jakovác ${ }^{\mathrm{a}}$ \\ Institute of Physics, BME Technical University, H-1111 Budapest, Hungary
}

\begin{abstract}
Transport coefficients, in particular the shear viscosity to entropy density ratio are studied in systems where the small-width quasiparticle assumption is not valid. It is found that $\eta / s$ has no universal lower bound, the minimal value depends on the system and the temperature, and can be even zero. We construct models where the $1 / 4 \pi$ conjectured bound is violated.
\end{abstract}

\section{Introduction}

Recent results from RHIC [1] suggest that hadronic matter near $T_{c}$ behaves as an almost ideal fluid [2]. Matter with small viscosity is very hard to describe theoretically, based on model calculations. The main reason is that the usual perturbative approach expands the theory around the free gas limit, where, by construction, the elementary excitations are in the ballistic regime with infinite mean free path. Since all transport coefficients are proportional to the mean free path, we obtain infinite results for them in the unperturbed regime. With formulae we can state that $\eta \sim 1 / \sigma \sim 1 / g^{4} \times \operatorname{logs}$ in QCD, where $\sigma$ is the cross section, $g$ is the coupling constant. In fact transport description assumes diffusion, the transport coefficients are diffusion constants. If we enforce a diffusive description to the ballistic regime, we obtain infinite diffusion constant.

Therefore the small shear viscosity coefficient measured at RHIC means that the elementary excitations of the matter after the collision have very short mean free path. Short mean free path means short lifetime, large width, and so a not a small-width quasiparticle system. If we still want to enforce an interacting free gas picture, it can be done only with very strong interactions. To describe such a system requires new approaches.

A possible exact method would be using lattice Monte Carlo (MC) simulations. One can measure $\left\langle T_{12}(x) T_{12}(0)\right\rangle$ correlator on lattice in Euclidean time, and use exact integral relations [3] to determine the commutator $C(x)=$ $\left\langle\left[T_{12}(x), T_{12}(0)\right]\right\rangle$. One has to invert this relation based on only a discrete set of time arguments. To make it feasible one has to use prior knowledge on the result (eg. $C(\omega>0)>0)$, and incorporate it via the Maximal Entropy or similar method. Then one can extract the shear viscosity from the Kubo formula as $\eta=\lim _{\omega \rightarrow 0} \frac{C(\omega, \mathbf{k}=0)}{\omega}$. The hard point of this analysis is that the Euclidean correlation function depends only very weakly on the derivative of $C$ at zero frequency, ie. viscosity [4]. This means that one has to have large systematical uncertainties. Nevertheless one

\footnotetext{
a e-mail: jakovac@phy.bme.hu
}

can give estimates as $\eta / s=0.102(56)$ at $T=1.24 T_{c}$ [3], where the uncertainty reflects the statistical error.

Another method to treat strongly interacting models to find a dual description with small coupling. For QCD such dual theory is not known, the "closest" theory where a dual theory can be constructed is the $\mathcal{N}=4$ super Yang-Mills (SYM) theory. If the coupling and the number of colors are infinite $\left(N_{c} \gg 1\right.$ and $\left.\lambda=g^{2} N_{c} \gg 1\right)$, then the dual model is a five dimensional gravity with AdS spacetime. Then, from graviton scattering, one can obtain the shear viscosity coefficient and $\eta / s$, the latter turns out to be $1 / 4 \pi$ [5]. For a wide class of theories this is a lower bound [6].

Although strictly speaking there are no exact perturbative methods to treat the strongly interacting matter, one can give arguments to estimate $\eta / s$ [7]. This argumentation uses the quasiparticle picture: there $\eta \sim \epsilon \tau$ and $s \sim n$ where $\epsilon$ is the energy density, $\tau$ is the quasiparticle lifetime and $n$ is the particle density. In this case $\eta / s \sim E \tau$ where $E$ is the particle energy. For quasiparticles $E \geq \Delta E$, and so $\eta / s \geq \Delta E \tau \gtrsim \hbar$. Since the so obtained value is close to the one given by the $\mathcal{N}=4$ SYM at large couplings, it is tempting to interpret $1 / 4 \pi$ as a universal lower bound for any existing matter.

This strong statement has been discussed a lot in the recent literature, and there are counterarguments given against its generic validity. In the $5 \mathrm{D}$ gravity models, one can construct higher curvature and dilaton models [8] where the $1 / 4 \pi$ is no more a lower bound. An explicit counterexample was constructed in [9], where the authors use a multicomponent model where the mixing entropy can tune the $\eta / s$ to zero.

As the argumentation of [7] is concerned, it is strongly based on a quasiparticle picture. In an interacting model the spectral function always contains other structures than the quasiparticle peak, and these can change the conclusions based on quasiparticles. For example the presence of a multiparticle continuum decreases considerably the $\eta / s$ ratio [10].

From the experimental side, one can analyze the anisotropy of the created particles, in particular the $v_{2}$ coefficient. From hydro fits one can readily give an upper

This is an Open Access article distributed under the terms of the Creative Commons Attribution-Noncommercial License 3.0, which permits unrestricted use, distribution, and reproduction in any noncommercial medium, provided the original work is properly cited. 
bound for the $\eta / s$ ratio [11] $(\eta / s<0.16)$, but it is much more difficult to present a lower bound. Taking into account the quadratic $p_{T}$ dependence of $\eta / s$ [12], the true value can be very close to the conjectured lower bound [13].

In other, more controllable experiments it is found [14] that supercritical fluids may have very small $\eta / s$ (or physically equivalent) ratio.

All these speculations reflect the fact that the status of the minimum of the $\eta / s$ ratio is still not clarified. The goal of the present work is to determine, what can be said about this ratio in a generic model, without assuming a smallwidth quasiparticle picture. The generic model will be defined by giving the energy levels of the system (density of states, DoS). For a more detailed discussion see Ref.s [15] and [16].

The paper is organized as follows. In Section 2 we analyze the $\eta / s$ ratio in a generic model and determine the minimum of it. In Section 3 we consider some models where the conjectured $1 / 4 \pi$ bound can be violated. We close the discussion with conclusions in Section 4.

\section{Transport and entropy in generic models}

A definite difference between the quasiparticle DoS and a generic one is the presence of continuum, ie. there is no dispersion relation, at fixed spatial momentum the system has still a lot of energy levels. To give an account for this property we define

$$
\sum_{n}|n\rangle\left\langle n\left|=V \sum_{Q} \int \frac{d^{4} p}{(2 \pi)^{4}} \varrho_{Q}(p)\right| p, Q\right\rangle\langle p, Q|
$$

where $\varrho$ denotes the DoS also called spectral function, $Q$ denotes conserved quantities (quantum channel), $p=$ $\left(p_{0}, \mathbf{p}\right)$ is the total energy-momentum of the state. $V$ is the volume, and we will use finite volume normalization for the states.

In order to be able to study the transport coefficients in general, we define the correlators $C$ for generic conserved current $J$, and the transport coefficient $D$ by the Kubo formula (linear response theory):

$$
C(x)=\left\langle\left[J_{i}(x), J_{i}(0)\right]\right\rangle \quad D=\lim _{\omega \rightarrow 0} \frac{C(\mathbf{k}=0, \omega)}{\omega},
$$

for the shear viscosity $D=\eta$ and $J_{i} \rightarrow T_{12}$. The expectation value is taken in equilibrium:

$$
\langle A\rangle=\frac{1}{\mathcal{Z}} \operatorname{Tr} e^{-\beta H} A, \quad \mathcal{Z}=\operatorname{Tr} e^{-\beta H}=e^{-\beta F},
$$

where $F$ is the free energy.

To find out a generic formula for the transport coefficients we insert an energy-momentum eigenbasis into the formula, and use the fact that translation is generated by $P_{\mu}$ energy-momentum operator as $J_{i}(x)=e^{i P x} J_{i}(0) e^{-i P x}$. Then we find

$$
\begin{aligned}
& C(x)=\frac{1}{\mathcal{Z}} \sum_{n}\left\langle n\left|e^{-\beta H}\left[J_{i}(x), J_{i}(0)\right]\right| n\right\rangle= \\
& =\frac{1}{\mathcal{Z}} \sum_{n, m}\left(\left\langle n\left|e^{-\beta H} J_{i}(x)\right| m\right\rangle\langle m| J_{i}(0)\right]|n\rangle- \\
& \left.\left.\left\langle m\left|e^{-\beta H} J_{i}(0)\right| n\right\rangle\langle n| J_{i}(x)\right]|m\rangle\right)= \\
& =\frac{1}{\mathcal{Z}} \sum_{n, m} e^{i\left(P_{n}-P_{m}\right) x}\left(e^{-\beta E_{n}}-e^{-\beta E_{m}}\right)\left|\left\langle n\left|J_{i}(0)\right| m\right\rangle\right|^{2} .
\end{aligned}
$$

By inserting the DoS we find

$$
\begin{aligned}
C(x)= & \frac{V^{2}}{\mathcal{Z}} \sum_{\mathcal{K}, Q} \int \frac{d^{4} k}{(2 \pi)^{4}} \frac{d^{4} q}{(2 \pi)^{4}} \varrho_{\mathcal{K}}(k) \varrho_{Q}(q) e^{i(k-q) x} \times \\
& \times\left(e^{-\beta k_{0}}-e^{-\beta q_{0}}\right)\left|\left\langle k, \mathcal{K}\left|J_{i}(0)\right| q, Q\right\rangle\right|^{2},
\end{aligned}
$$

After Fourier transformation, with $\mathbf{p}=0$ zero spatial momentum

$$
\begin{aligned}
& C(\omega, \mathbf{p}=0)=\frac{V^{2}}{\mathcal{Z}} \sum_{\mathcal{K}, Q} \int \frac{d^{4} k}{(2 \pi)^{4}} \varrho_{\mathcal{K}}(k) \varrho_{Q}\left(\mathbf{k}, k_{0}+\omega\right) \times \\
& \times\left(e^{-\beta k_{0}}-e^{-\beta\left(k_{0}+\omega\right)}\right)\left|\left\langle\mathbf{k}, k_{0}, \mathcal{K}\left|J_{i}(0)\right| \mathbf{k}, k_{0}+\omega, Q\right\rangle\right|^{2} .
\end{aligned}
$$

We can take into account that the current cannot change the quantum numbers without changing the energy or momentum, then the diffusion constant reads

$$
D=\beta \frac{V^{2}}{\mathcal{Z}} \sum_{\mathcal{K}} \int \frac{d^{4} k}{(2 \pi)^{4}} \varrho_{\mathcal{K}}^{2}(k) e^{-\beta k_{0}}\left|\left\langle k, \mathcal{K}\left|J_{i}(0)\right| k, \mathcal{K}\right\rangle\right|^{2} .
$$

In the free theory the expectation value of the current can be written in volume normalization as $\left\langle k\left|J_{i}\right| q\right\rangle=q k_{i} /\left(k_{0} V\right)$, where $q$ is the charge carried by the current; for the shear viscosity $q \rightarrow k_{j}$. In general the current matrix element is proportional to the velocity $v_{i}=k_{i} / k_{0}$, and in volume normalization it is inversely proportional to the volume. So the generic formula is proportional to the free case, and we can write

$$
\left|\left\langle k, \mathcal{K}\left|J_{i}(0)\right| k, \mathcal{K}\right\rangle\right|^{2}=\mathcal{J}_{\mathcal{K}}^{2}\left(k^{2}\right)\left(\frac{q k_{i}}{k_{0} V}\right)^{2},
$$

where $\mathcal{J}_{\mathcal{K}}\left(k^{2}\right)$ is a nonperturbative correction factor. Then

$$
D=\frac{\beta}{\mathcal{Z}} \sum_{\mathcal{K}} \int \frac{d^{4} k}{(2 \pi)^{4}} \frac{q^{2} k_{i}^{2}}{k_{0}^{2}} e^{-\beta k_{0}}\left(\varrho_{\mathcal{K}}(k) \mathcal{J}_{\mathcal{K}}\left(k^{2}\right)\right)^{2} .
$$

As a final step we can average over the spatial angular dependence. This gives $\overline{k_{i}^{2}}=\mathbf{k}^{2} / 3$ and $\overline{k_{x}^{2} k_{y}^{2}}=\left(\mathbf{k}^{2}\right)^{2} / 15$. Then the shear viscosity reads

$$
\eta=\frac{\beta}{15 \mathcal{Z}} \sum_{\mathcal{K}} \int \frac{d^{4} k}{(2 \pi)^{4}} \frac{\left(\mathbf{k}^{2}\right)^{2}}{k_{0}^{2}} e^{-\beta k_{0}}\left(\varrho_{\mathcal{K}}(k) \mathcal{J}_{\mathcal{K}}\left(k^{2}\right)\right)^{2} .
$$

After having defined the shear viscosity, we can try to give a formula for the entropy density. This is, however, 
a conceptually more difficult task. The point is that the entropy density, being a statistical concept, is not sensible in the microscopic theory. Indeed, if we consider the volume dependence of the free energy defined from the partition function in eq. (3), at small volumes it can show any volume dependence (usually grows very fast), only at large volumes will it be proportional to the volume. There is a "crossover" size $L$, where statistical treatment starts to be sensible. This size corresponds to the coarse graining scale, beyond that two neighboring volume elements (grains) of linear size $L$ interact dominantly through the surface, which modify the total energy of them only weakly. This means that interactions are effectively cut off at scale $L$, while statistics start to be valid above $L$. The value of $L$ can be read off the linear size of the cross section: for a strongly interacting theory $L$ is large, a weakly interacting theory has small $L$.

Having said that we choose a volume $V=L^{3}$ to define the free energy density and entropy density as

$f=-\frac{T}{L^{3}} \ln \left(1+L^{3} \sum_{\mathcal{K}} \int \frac{d^{4} k}{(2 \pi)^{4}} \varrho \mathcal{K}(k) e^{-\beta k_{0}}\right), \quad s=-\frac{\partial f}{\partial T}$.

So finally we obtain

$$
\frac{\eta}{s}=\frac{\frac{\beta}{15 \mathcal{Z}} \sum_{\mathcal{K}} \int \frac{d^{4} k}{(2 \pi)^{4}} \frac{\left(\mathbf{k}^{2}\right)^{2}}{k_{0}^{2}} e^{-\beta k_{0}}\left(\mathcal{J}_{\mathcal{K}}(k) \varrho_{\mathcal{K}}(k)\right)^{2}}{\frac{\partial}{\partial T} \frac{T}{L^{3}} \ln \left(1+L^{3} \sum_{\mathcal{K}} \int \frac{d^{4} k}{(2 \pi)^{4}} \varrho_{\mathcal{K}}(k) e^{-\beta k_{0}}\right)} .
$$

After having found this formula we can ask whether there is a lower bound in this formula. The generic analysis can be found in [16], but the generic form can be easily understood. For that consider the small entropy case: then in the $\log$ in the denominator the 1 will be dominant, and we can write

$$
\left.\frac{\eta}{s}\right|_{\text {small } s}=\frac{\frac{\beta}{15 \mathcal{Z}} \sum_{\mathcal{K}} \int \frac{d^{4} k}{(2 \pi)^{4}} \frac{\left(\mathbf{k}^{2}\right)^{2}}{k_{0}^{2}} e^{-\beta k_{0}}\left(\mathcal{J}_{\mathcal{K}}(k) \varrho \mathcal{K}(k)\right)^{2}}{\sum_{\mathcal{K}} \int \frac{d^{4} k}{(2 \pi)^{4}} \varrho_{\mathcal{K}}(k)\left(1+\beta k_{0}\right) e^{-\beta k_{0}}} .
$$

From here we see that the numerator is proportional to $\varrho^{2}$ while the denominator by $\varrho$. If $\varrho$ is zero almost everywhere and very large at some point - this is the small width quasiparticle limit,$- \varrho^{2}$ is even larger at these point, and the result will be large. Therefore in small width quasiparticle limit $\eta / s$ is large. On the other hand, is $\varrho$ is small everywhere, then $\varrho^{2}$ is even smaller, and the result is small. This means that small $\eta / s$ can be expected in system where there are no long-lived quasiparticles!

To be more quantitative we rewrite our formula as:

$$
\left.\frac{\eta}{s}\right|_{\text {small } s}=\frac{\left\langle\left\langle\bar{\varrho}^{2}\right\rangle\right\rangle}{\langle\langle\bar{\varrho}\rangle\rangle}
$$

where the rescaled DoS reads as

$$
\bar{\varrho} \mathcal{K}(k)=\frac{\beta}{15 \mathcal{Z}} \frac{\left(\mathbf{k}^{2}\right)^{2}}{k_{0}^{2}\left(1+\beta k_{0}\right)} \mathcal{J}_{\mathcal{K}}^{2}(k) \varrho \mathcal{K}(k)
$$

and the averaging is interpreted as $\langle\langle\ldots\rangle\rangle=\mathcal{N}^{-1} \int d A(\ldots)$ where the integration measure is

$$
d A=15 \mathcal{Z} T \sum_{\mathcal{K}} \frac{d^{4} k}{(2 \pi)^{4}} \frac{k_{0}^{2}\left(1+\beta k_{0}\right)^{2}}{\left(\mathbf{k}^{2}\right)^{2}} \mathcal{J}_{\mathcal{K}}^{-2}(k) e^{-\beta k_{0}},
$$

and the normalization factor reads

$$
\mathcal{N}=15 \mathcal{Z} T \sum_{\mathcal{K}} \int \frac{d^{4} k}{(2 \pi)^{4}} \frac{k_{0}^{2}\left(1+\beta k_{0}\right)^{2}}{\left(\mathbf{k}^{2}\right)^{2}} \mathcal{J}_{\mathcal{K}}^{-2}(k) e^{-\beta k_{0}}
$$

Now we can apply the Schwarz inequality: $\left\langle\left\langle\bar{\varrho}^{2}\right\rangle\right\rangle \geq$ $\langle\langle\bar{\varrho}\rangle\rangle^{2}$, and we see:

$$
\left.\frac{\eta}{s}\right|_{\text {small } s} \geq \mathcal{N}^{-1} s
$$

What we see is that, although $\eta / s$ has a minimum, but it is no universal (like $1 / 4 \pi$ ), it is model- and environmentdependent. The minimal value can even be zero by tuning the system to reach $\mathcal{N} \rightarrow \infty$ (cf. [9]), or go to $s \rightarrow 0$ ie. to zero temperature.

A more detailed analysis, including also systems with large entropy density shows [16] that

$$
\left.\frac{\eta}{s}\right|_{\text {minimum }}=\frac{\mathcal{F}\left(L^{3} s\right)}{N_{Q}(L T)^{4}}
$$

where $\mathcal{F}(x) \sim x$ for small $x$ and $\sim e^{x} / x$ for large $x$, and $N_{Q}$ is the effective number of quantum channels (particle species).

\section{Model calculations}

After having found the minimal value, we can try to construct physically relevant models where this minimum can be reached. To this end we make some simplifications in the above calculation. First, we use a generalized quasiparticle systems, and substitute the DoS by the quasiparticle spectral function. Then the free energy reads for bosonic or fermionic systems

$$
f=T \int \frac{d^{4} k}{(2 \pi)^{4}} \varrho_{Q P}(k)(\mp) \ln \left(1 \pm e^{-\beta k_{0}}\right) .
$$

A second simplification is that we omit the radiative corrections from the current matrix elements, ie. $\mathcal{J}_{\mathcal{K}}(k)=1$. Third, we take only one quantum channel. In this way the "reduced" viscosity coefficient reads

$$
\bar{\eta}=\frac{\beta}{15} \int \frac{d^{4} k}{(2 \pi)^{4}} \frac{\left(\mathbf{k}^{2}\right)^{2}}{k_{0}^{2}} e^{-\beta k_{0}} \varrho_{Q P}^{2}(k) .
$$

\subsection{Small width case}

To check the validity of our approximations, we apply it to a small-width quasiparticle case. Assume that the lowest lying states can be approximated with Breit-Wigner form

$$
\varrho_{Q P}(k)=\frac{2 \Gamma}{\left(k_{0}-\varepsilon_{k}\right)^{2}+\Gamma^{2}},
$$


where $\varepsilon_{k}$ is the dispersion relation.

In the small width limit $\varrho(q)^{2} \approx \frac{2}{T} 2 \pi \delta\left(q_{0}-\varepsilon_{q}\right)$. The formulae (20) and (21) can be calculated [16] with the result

$$
\frac{\bar{\eta}}{s}=\frac{T}{\Gamma} f\left(\frac{m}{T}\right),
$$

where the function $f$ depends on the dispersion relation, and $m$ is the mass scale. For example for $\varepsilon_{k}=k, f=$ $540 / \pi^{4}$ for bosons, $f=4320 /\left(7 \pi^{4}\right)$ for fermions; if $\varepsilon_{k}=$ $m+\frac{k^{2}}{2 m}, f=30 \pi T / m$.

If we consider a massless theory (eg. conformal field theory) then the only scale is $T$, and so $\Gamma \sim T$. This means that $\bar{\eta} / s=$ constant. The lower limit of this constant may come from infinite coupling, $1 / 4 \pi$.

In the massive case the width of the particle should come from scattering process, therefore at low temperature we expect $\Gamma \sim e^{-M / T}$, where $M$ is the energy of the lowest scattering state. In this case $\bar{\eta} / s \sim T e^{M / T} \stackrel{T \rightarrow 0}{\rightarrow} \infty$.

This means that in the small width quasiparticle case there is a lower bound, which comes from the massless theory.

\subsection{Broad spectral function}

For an opposite case consider a flat spectral function:

$$
\varrho_{Q P}\left(k_{0}, k\right)=\frac{2 \pi}{E_{2}-E_{1}} \Theta\left(E_{1}<k_{0}<E_{2}\right)
$$

which is a step function, where $E_{1,2}(k)=\sqrt{k^{2}+m_{1,2}^{2}}$. At small temperatures $\left(T<m_{1}\right)$ we find [16]

$$
\frac{\eta}{s}=6 \pi \frac{T}{m_{2}-m_{1}}
$$

This formula suggests that by broadening the energy distribution, the viscosity to entropy density ratio has no lower bound, it can be made vanish. This is exactly the message of the generic analysis: since $\varrho$ is normalized, broadening means small values, and so $\varrho^{2} \ll \varrho$ everywhere. This results in the smallness of $\eta / s$ ratio.

\subsection{System with zero mass excitations}

Although the above example may be not physical, there are examples where the spectral function has no isolated quasiparticle peak, but it starts with a continuum. This is the case when an interacting system contains zero mass particles: then the multiparticle cut start directly from the quasiparticle peak. The interpretation is that a charged object is always surrounded by soft gauge bosons. For example in QED at one loop level the electron spectral function [17], up to log corrections, is proportional to $\varrho_{Q P}(k) \sim$ $\left(k^{2}-m^{2}\right)^{-1}$, ie. it is divergent at the mass shell, and fall as $1 /\left(\omega-\varepsilon_{k}\right)$. By Bloch-Nordsieck resummation it changes to $1 /\left(\omega-\varepsilon_{k}\right)^{1-b}$, where $b$ is the beta function.
In order $\varrho$ to be normalizable, the singular behavior at the mass shell must be smoothed out by some physical process (cf. also [18]). Near the threshold (which is the dominant regime at low temperatures) we approximate

$$
\varrho_{Q P}(q)=C q_{0} \Theta(q-M)\left(q^{2}-M^{2}\right)^{w},
$$

where $w \geq-1$ for normalizability, and $C$ is a constant, its dimension is $[C]=[E]^{-2(1+w)}$. Since $\eta \sim C^{2}$ and $f \sim C$, therefore $C$ remains in the ratio. In the massive and massless case we find

$$
\frac{\bar{\eta}}{S} \sim C M^{w} T^{2+w} \quad \text { and } \quad C T^{2(1+w)} \stackrel{T \rightarrow 0}{\longrightarrow} \quad 0 .
$$

In the limiting $w=-1$ case the $\eta / s$ is constant, this is the case in some conformal models.

This analysis suggests that in a system with zero mass excitations, but normalizable spectral functions the $\eta / s$ ratio should be vanishing at very small temperatures.

\section{Conclusions}

In this paper we discussed the behavior of the the shear viscosity to entropy ratio in systems where the small-width quasiparticle approximation is not necessarily true. We have found that, although $\eta / s$ has a lower bound in each given system at a given temperature, but this bound is system- and temperature dependent; for small entropy case we found for the minimum $\sim \frac{s}{N_{Q} L T^{4}}$ where $L$ is the interaction length and $N_{Q}$ is the number of effective quantum channels (particle species). This minimum can be smaller than $1 / 4 \pi$. This fact was demonstrated by constructing models with this property: if the spectral function does not go to an isolated quasiparticle peak at zero temperature, which is a natural setup in systems with zero mass excitations, then $\eta / s \rightarrow 0$ at zero temperature.

\section{Acknowledgment}

This work was supported by the Hungarian Science Fund (OTKA) K68108.

\section{References}

1. S. S. Adler et al. [PHENIX Collaboration], Phys. Rev. Lett. 91 (2003) 182301 [nucl-ex/0305013]. J. Adams et al. [STAR Collaboration], Phys. Rev. Lett. 92 (2004) 052302 [nucl-ex/0306007].

2. E. Shuryak, Prog. Part. Nucl. Phys. 53 (2004) 273

3. F. Karsch and H. W. Wyld, Phys. Rev. D 35 (1987) 2518; H. B. Meyer, Phys. Rev. D 76 (2007) 101701 [0704.1801 [hep-lat]]

4. P. Petreczky, J. Phys. G 35 (2008) 044033 [arXiv:0710.5561 [nucl-th]].

5. P. Kovtun, D.T. Son, A.O. Starinets JHEP 0310 (2003) 064. 
6. A. Buchel, R.C. Myers, M.F. Paulos, A. Sinha, Phys. Lett. B 669 (2008) 364; M. Haack, A. Yarom, Nucl. Phys. B 813 (2009) 140 [arXiv:0811.1794]; R.C. Myers, M.F. Paulos, A. Sinha, Phys. Rev. D 79 (2009) 041901 [arXiv:0806.2156]

7. P. Danielewicz, M. Gyulassy, Phys. Rev. D 31 (1985) 53; P. Kovtun, D.T. Son, A.O. Starinets Phys. Rev. Lett. 94 (2005) 111601

8. P. Kats and P. Petrov, JHEP 01 (2009) 044 [arXiv:0712.0743]; A. Buchel, R.C. Myers and A. Sinha, JHEP 03 (2009) 084 [arXiv:0812.2521]; R.-G. Cai, Z.-Y. Nie, N. Ohta and Y.-W. Sun, Phys. Rev. D 79 (2009) 066004 [arXiv:0901.1421]

9. A. Cherman, T. D. Cohen, and P. M. Hohler, JHEP 02 (2008) 026 [arXiv:0708.4201]

10. J. Noronha-Hostler, J. Noronha and C. Greiner, Phys. Rev. Lett. PRL 103 (2009) 172302 [arXiv:0811.1571]

11. P. Romatschke, U. Romatschke, Phys. Rev. Lett. 99 (2007) 172301

12. D.A. Teaney, arXiv:0905.2433

13. R.A. Lacey, A. Taranenko, R. Wei, arXiv:0905.4368

14. J. Liao and V. Koch, Phys. Rev. C 81 (2010) 014902 [arXiv:0909.3105]

15. A. Jakovac and D. Nogradi, arXiv:0810.4181; A. Jakovac, arXiv:0901.2802

16. A. Jakovac, Phys. Rev. D. 81 (2010) 045020 [arXiv:0911.3248]

17. N.N. Bogoljubov and D.V. Shirkov, Introduction to the Theory of Quantized Fields (1980, John Wiley \& Sons, Inc.)

18. T.S.Biro, P.Levai, P.Van, J.Zimanyi, Phys. Rev. C 75 (2007) 034910 